\title{
Oral Corticosteroid Use for the Treatment of Chronic Eosinophilic Disease: A Patient's and His Physician's Experience
}

\author{
Mitchell Silva $\cdot$ Florence Roufosse
}

Received: June 26, 2019 / Published online: August 31, 2019

(C) The Author(s) 2019

\begin{abstract}
This article, coauthored by a patient with eosinophilic granulomatosis with polyangiitis (EGPA) initially presenting as severe eosinophilic asthma and his physician-specialist, discusses the use and management of oral corticosteroid (OCS) treatment. It also considers the importance of early diagnosis of a rare disease and patient education. The patient describes his journey from progressive worsening of asthma and eventual diagnosis of EGPA to long-term OCS treatment and then participation in a clinical trial for this rare disease, involving the introduction of targeted biologic therapy with OCS tapering. The physician describes the importance of patient referral to obtain a correct diagnosis and optimal maintenance treatment, the balance between risk of adverse events associated with long-term OCS use and benefits of disease control, and various aspects of patient participation in clinical trials.
\end{abstract}

Enhanced Digital Features To view enhanced digital features for this article go to https://doi.org/10.6084/ m9.figshare.9617381.

M. Silva

Esperity, Brussels, Belgium

e-mail: mitchell@esperity.com

F. Roufosse $(\bowtie)$

Department of Internal Medicine, Hôpital Erasme,

Université Libre de Bruxelles, Brussels, Belgium

e-mail: froufoss@ulb.ac.be
Finally, the patient describes the role of continual patient education in the management of disease and OCS treatment. These considerations can apply to all chronic inflammatory diseases requiring maintenance OCS treatment. Funding: AstraZeneca.

Keywords: Asthma; EGPA; Eosinophilic granulomatosis with polyangiitis; OCS; OCS tapering; Oral corticosteroids; Patient education; Added; Severe eosinophilic asthma

\section{INTRODUCTION}

Careful use of oral corticosteroid (OCS) therapy should go hand in hand with a monitoring strategy and lifestyle modifications to reduce the risk of adverse events (AEs). This involves patient education and transparent communication between patients and physicians, aimed at engaging patients in their own care trajectories. For an increasing number of chronic inflammatory disorders, given the fact that more innovative treatments such as biologics might offer alternatives to OCS, shared decision-making plays an increasingly important role in treatment choice. Knowing more about the perception of AEs associated with long-term OCS treatment might impact the process of shared decision-making. Patients' major concerns regarding OCS use are weight gain, Cushingoid 
appearance, osteoporosis, and cataracts [1]. Negative perceptions regarding these AEs can influence medication adherence and consequently impact disease control. In addition, OCS-associated AEs with which patients are less familiar, but that significantly affect health, such as increased risk of diabetes, high blood pressure, etc., could further impact patients' decision-making if they were better informed. Health literacy also influences perceived control, as shown with asthma, in which improving patients' understanding of the disease can improve their health-related quality of life [2]. This raises the question as to what extent patient education is needed from both the patients' and physicians' perspectives when the patients receive maintenance OCS treatment.

In this article, a case will be presented in which concerns relating to long-term OCS use are addressed by a patient and his physician in the specific clinical setting of severe eosinophilic asthma progressing to eosinophilic granulomatosis with polyangiitis (EGPA). Unraveling the key take-home messages from these two perspectives could lead to better communication between patients and physicians, and could eventually lead to better disease control and reduced risk and burden of AEs related to OCS use.

\section{Compliance with Ethics Guidelines}

This article does not contain any new studies with human or animal participants performed by any of the authors.

\section{THE PATIENT: CONTEXT AND BACKGROUND}

My journey as a patient started more than 10 years ago at the age of 29 , when I was diagnosed with severe eosinophilic asthma with a suspicion of Churg-Strauss syndrome, now referred to as EGPA [3]. I was formally diagnosed with EGPA 6 years later, 13 years after my first asthma symptoms occurred. The actual diagnosis of severe asthma was the result of a visit to the emergency department at my local hospital, after I experienced general malaise, night sweats, asthma symptoms, and immense chest pain, especially when lying on my back, over a period of months. The frequency and intensity of my symptoms increased gradually over time, and I was not very aware of the severity until I ended up in quarantine (to exclude a potential tuberculosis infection) for 2 weeks before finally receiving a diagnosis of EGPA and starting maintenance OCS treatment. Looking back, for at least a year before the EGPA diagnosis, my quality of life had been extremely bad. I had had frequent doctor visits but no medical improvement or further investigations.

After my diagnosis of severe eosinophilic asthma, and knowing about potential new biologic treatments, I searched for a physician who was recruiting for a clinical trial. After being accepted into the trial, I followed an OCS dosage-reduction scheme aimed at reducing OCS dosage as much as possible while maintaining disease control without compromising health risks.

\section{THE PHYSICIAN: CONTEXT AND BACKGROUND}

As an internist involved in the management of hypereosinophilic disorders for 20 years, I have had the opportunity to conduct translational research to address key clinical questions using biological samples obtained with consent from the patients I see in the clinic. The scientific observations made in the context of these research activities have been published, and consequently our institution has become a referral center for rare eosinophilic disorders, which has resulted in increased recruitment of patients. Importantly, this expertise and recruitment capability are attractive for pharmaceutical companies that are developing eosinophil-targeting drugs with more selective mechanisms of action, and therefore less toxicity, than OCS and the classic drugs that alter the immune response. In the setting of rare diseases, these companies often allow participants to continue the new drug in the setting of an open-label extension or on a compassionate use basis after trial completion. Having the 
opportunity to enroll patients in such trials is very satisfying, both because it is a way of actively participating in the process of obtaining approval for new targeted treatments and because it is possible to offer one's patients truly novel agents that allow OCS dosage reduction.

\section{THE PATIENT JOURNEY NARRATIVE FROM THE PHYSICIAN AND PATIENT PERSPECTIVES}

\section{Physician Perspective on the Patient Journey}

Delayed diagnosis is extremely common in rare chronic inflammatory diseases such as EGPA. Potential consequences of delayed diagnosis include progressive decline of quality of life with social and professional implications, mood disorders (anxiety and depression) with associated somatization that further obscure diagnosis, and development of irreversible complications of uncontrolled inflammation.

Factors that contribute to this delay are diverse. From the patient's perspective, denial of symptoms and difficulties in acknowledging the possibility of a serious chronic disease are common, especially in the setting of a busy lifestyle in which raising a family, working, and functioning socially fulfill the human need for a meaningful life. Only when a patient feels that these functions are menaced by increasingly incapacitating symptoms will he or she seek health care. It may take years before the patient establishes a medical contact and additional years before the patient is actually "matched" with the "right" physician who can both name the disease and offer the most appropriate (i.e., most effective and less toxic) treatment. Typically, as described above, the patient realizes the chronicity of his or her ailment only in retrospect, when medical history is established through a dialogue with the physician and a diagnosis is made.

From the physician's perspective, failure to recognize the limits of one's knowledge and experience may delay referral of patients with rare disorders such as EGPA. These limits should be accepted with humility on the physician's behalf and leniency on the patient's behalf. The patient-physician relationship, ideally based on mutual respect and benevolence on behalf of the latter, should address the question regarding the adequacy of the match upfront.

\section{Patient Perception of OCS Use Prior to Diagnosis}

Prior to being diagnosed with EGPA, I was prescribed short-term OCS treatment courses, often in combination with antibiotics, when my asthma symptoms worsened. This gave temporary symptom relief, often for a prolonged period, until the next exacerbation occurred. At those times, the communication I received concerned the appropriate OCS dosage with a clear message to follow the suggested tapering regimen to avoid possible complications. I did not ask many questions at that time about OCS because of the treatment's high effectiveness and did not think about the possibility of a more severe disease. My understanding of asthma at that time was that it was a rather controlled and manageable disease, not substantially impacting quality of life. This is because asthma is so widespread, and I knew so many other people receiving short-acting $\beta_{2}$ agonists. I was prescribed a standard treatment of local inhaled corticosteroids (ICS) for longterm use. Contrary to the OCS, adherence to ICS use was not emphasized. I often used ICS only when experiencing symptoms. However, the literature has stated that daily use of low-dose ICS is important for asthma control [4].

I did not pay much attention to or have any burning questions about the possible long-term AEs of corticosteroids at that time. There was no communication about long-term "attention points" by my primary physician, pulmonologist, or pharmacist. The only thing that resonated with me was that tapering is important when receiving OCS to avoid adrenal gland problems, and, for ICS, that rinsing the mouth is important to avoid possible fungal infection in the oral cavity. Looking back at my patient journey, I absolutely would have benefited from education about my condition and its 
treatment, namely about paying attention to the frequency of asthma worsening and OCS courses. Had I been aware that increasing use of corticosteroids over time should lead me to visit a specialist and re-evaluate the disease, I may have avoided being hospitalized in very bad shape with pulmonary infiltrates.

\section{Physician Perspective on Repeated Courses of OCS for Chronic Inflammatory Diseases Characterized by Exacerbations}

Severe eosinophilic asthma is one of many immune-mediated diseases that may behave relatively well during a given period of time and then flare suddenly, with acute and severe symptoms impossible to ignore. When asymptomatic, patients are more concerned about life activities than their health and typically skip, then forget entirely, their maintenance ICS regimen. During flares, patients seek urgent medical care and often receive short-term instructions on how to manage the flare, namely with regard to OCS treatment, tapering, and withdrawal. As the treatment duration is short, it makes little sense to give instructions on diet and other measures for preventing the occurrence of AEs associated with OCS use.

Given the acute nature of the symptoms and the usually rapid response to treatment, patients resume daily activities and do not worry much about the incident, nor do they necessarily visit a specialist when they are feeling well to make sure there are no implications with regard to long-term health. In many cases, such patients do not even see the same physician for each flare, meaning no one (neither patient nor physician) is keeping track of whether exacerbation frequency and/or the cumulative amount of ICS and OCS absorption are increasing over time. An increase in frequency and severity of flares and increased need for treatment are clear indicators that the disease is progressing and should be investigated thoroughly. The goals should be to refine the diagnosis and classification of the disease, to evaluate global disease severity, and to look ahead in terms of long-term disease control. This is the correct time to look at eosinophil counts and perform lung imaging. This is also the time to plan future treatment and consider maintenance therapy.

In the specific field of illnesses characterized by recurrent exacerbations, neglecting disease worsening may have a significant negative impact on patient health if such strategies are not adopted. On the one hand, the cumulative dosage (and therefore toxicity) of short-term OCS in the setting of repeated courses may actually exceed the dosage administered in the setting of continuous low-dosage therapy. On the other hand, letting the disease flare between courses of OCS may progressively lead to irreversible tissue damage.

\section{Patient Perception of OCS at Diagnosis of Severe Eosinophilic Asthma}

At diagnosis of severe eosinophilic asthma, the initial treatment consisted of methylprednisone $128 \mathrm{mg} \mathrm{IV}$, followed by a tapering regimen to OCS $32 \mathrm{mg}$ after 4 days. Within $24 \mathrm{~h}$ after the first IV administration, my symptoms significantly improved. At that moment, I started systemic OCS therapy, and have continued through today.

In general, patients have a very negative perception about OCS use, primarily driven by the physical AEs that are associated with OCS, such as weight gain, "moon face", increased facial hair growth, and fluid retention, among others. For most patients, less apparent AEs such as osteoporosis, diabetes, and hypertension can only be brought to their attention by education [5]. These topics were never addressed in depth by my health care provider at that time, but knowing about these possible risks, I paid attention and started taking calcium as well as vitamin D supplements and regularly measured my blood glucose concentrations. Being a good responder to OCS without apparent AEs related to OCS use, I accepted "the new normal" and felt I was in control of my health, not really feeling concerned about keeping the OCS dosage as low as possible to reduce longterm AEs because I felt well. In addition, I was not inclined to decrease my dosage because disease symptoms that significantly affect 
quality of life and sleep, such as nasal congestion and wheezing, recurred rapidly after attempts to taper treatment. It would be interesting to assess patient behavior in terms of severity and frequency of such symptoms for treatment dosage and involved risks with greater OCS dosage.

Knowing that OCS-sparing strategies exist, including strict monitoring and management of local (nasal and inhaled) OCS use, might have helped to trigger the right questions during consultations to achieve the lowest possible dosage. The question is when in the patient journey is this type of information best communicated to the patient, and how, in relation to the patient profile and disease acceptance?

\section{Physician Perception of Patient Education and Measures to Take When Introducing Maintenance OCS Therapy}

When disease progression and severity are such that maintenance therapy with OCS is necessary, the physician has an important responsibility regarding patient education and awareness about short-, medium-, and longterm toxicity. People differ in their education and access to information, and also in how they cope with their disease and how they regard physicians. They also differ in their tolerance of OCS and propensity to develop AEs. Because to date there are absolutely no features that help predict which patients will experience more or less severe toxicity, in all cases the physician must make a series of recommendations about diet and lifestyle, proactively investigate risk factors for complications of treatment, and initiate certain preventive measures, from the very start of treatment.

Important questions to tackle when starting OCS include: Does the patient ingest dairy products daily? Has the patient had an ulcer in the past? Does the patient take anti-inflammatory pain killers regularly (increasing the risk of ulcers when combined with OCS)? Does the patient have some form of regular physical activity that will naturally counteract the sarcopenia and osteoporosis that accompany OCS therapy? Is there a family history of diabetes, or has the patient had increased fasting glucose concentrations in the past? Patient habits and history may increase the risk of specific AEs of OCS use, which can, therefore, be anticipated and explored earlier and more frequently. It is important to know what their blood pressure, blood glucose concentrations, and bone density are before initiating treatment, as these can all be rapidly negatively impacted. Travel history and infection/vaccination status are also interesting to explore, as some infections may be reactivated or worsened with OCS treatment, and the immune response to vaccines will be better before the initiation of OCS.

It is much easier to adopt good habits from the start of treatment rather than having to correct/reverse behavior later. Patients should be informed that they may experience fluid retention and so limit salt intake for this reason. They should know that the sensation of "food craving" is common, and should anticipate this by preparing hypocaloric food that they enjoy and keeping it close at hand. If their lifestyles are sedentary, a few visits with a physiotherapist may be useful to educate them about how to prevent muscle atrophy. Finally, in addition to OCS pills, the physician should prescribe, at minimum, calcium and vitamin D supplements to prevent bone demineralization. For patients at high risk of ulcers, a proton pump inhibitor may be warranted. However, these additional pills increase patient perception that treatment is a burden, and the physician may feel hesitant to overwhelm the patient with a long list of prescriptions. These aspects interfere with the implementation of beneficial preventive measures.

The question of patient preference for disease-related discomfort (that is relieved by OCS) versus OCS toxicity is influenced by many patient-dependent factors, namely the importance of perceived AEs of OCS for a given patient and his or her prior experience (personal or close family and friends) with OCS toxicity. People who tolerate OCS very well in the short term, and who have never witnessed the potentially devastating effects of long-term OCS use, are likely to prefer the comfort of disease control achieved by OCS treatment, even at dosages greater than needed. In contrast, those 
who experience rapid physical changes with treatment and/or have seen someone dear become severely handicapped because of OCS use will be reluctant to pursue treatment and may prefer "putting up with" their disease. They are not necessarily aware that chronic inflammation is not less deleterious for tissues and organs than long-term OCS use.

These aspects should be discussed openly between patient and physician. Most physician-patient discussions are focused on disease, but it is essential that time be taken to discuss AEs associated with OCS use and to answer patient questions. More closely spaced outpatient visits at the beginning of maintenance treatment allow patients and physicians not only to assess the response to therapy and the AEs of OCS but also to address new questions about treatment as they arise.

\section{Patient Perception of Long-Term OCS Use After Start of Treatment}

I see two main challenges as a patient: first, being compliant, even after 10 years of using ICS, and, second, having clear objectives for lowering corticosteroid dosage. Coping with a disease is different for each individual, and often there is the risk that one would use his or her disease as an excuse to justify behavior or feelings/emotions, whether it is fatigue, anxiety, or nervous behavior. It is important to not be dragged down by such patterns and to be aware that concrete actions such as lifestyle modifications and goal-setting can help one cope with certain feelings and emotions that could negatively impact treatment management. Taking high dosages of OCS and overmedicating with ICS can trigger a perception of disease control and make one feel good, but, in fact, this is rather contrary in the case of overmedication. Before auto-medicating, it is important to be aware of the drivers of dosage changes. Do you desire a dosage change because of significantly increased symptoms? What are these symptoms? How long do you have them? What could be the consequences of these symptoms?
Since receiving a biologic treatment, I could reduce my OCS dosage by $30 \%$ on average. In general, my quality of life has also improved. I experience fewer exacerbations, although sometimes I get disappointed for just being a moderate responder and not a full responder. There are also other concerns related to OCS use, even at a decreased dosage, such as how my eyesight will evolve over time, what skin changes will occur, when I will experience signs of osteoporosis, whether the muscle weakness I feel is important, and what to do to counteract it. Over the years, I have learned not to panic: to take things as they come, be aware of and follow guidelines, and not allow my life to be driven by fear. However, I realize this is highly individual.

\section{Physician Perception of Long-Term OCS Use}

Once it appears clear that a given patient will be dependent on lifelong treatment to control chronic inflammatory disease, management of therapy should remain extremely dynamic. The "minimally effective OCS dosage" or "threshold of corticosteroid dependency" should be determined for each individual patient, to the milligram, on the basis of symptoms and diseasespecific objective features (such as blood eosinophil counts, lung function tests, etc.). Disease activity intrinsically fluctuates over time, with alternating periods of remission and exacerbations. It is well-established that patients with chronic inflammatory diseases are often overtreated to avoid the hassle of periodic exacerbations and the requirement of treatment adjustments. Nevertheless, the threshold of corticosteroid dependency may change over time, and it is always worthwhile to try to further decrease the dosage after the disease has been stable for a while. On the other hand, the disease may still exacerbate from time to time, requiring further temporary increases in OCS dosage and/or optimization of local (inhaled) treatment. In asthma treatment, there has been progress in the type of molecules found in inhalers and their delivery to the small airways, and adaptations in treatment should take these advances into account. 
If the dosage, observed toxicity, and/or discomfort related to the maintenance OCS dosage are judged excessive by the physician and/or the patient, it is time to consider the options in terms of corticosteroid-sparing agents. Depending on the disease, different types of immunosuppressive or immunomodulatory agents are available. In the field of severe eosinophilic asthma [6] and/or EGPA [7], several new molecules that act very specifically on a given molecule or cell type involved in disease have become available, and others are in development. The aim of these new drugs is to decrease the exposure to OCS through agents that have very targeted effects in the body and therefore less toxicity. If a patient is eligible for treatment with one of these new agents, attempts should be made to taper or withdraw maintenance OCS treatment.

\section{What the Clinical Trial Taught Me as a Patient on OCS Use}

The most important point that sticks with me is my reliance on OCS. Thanks to the clinical trial, I was in a very controlled and supported environment to go as low as possible, and I strictly followed a tapering schedule. Having the digital tools to enter a daily quality of life score and my daily OCS dosage added up to the perceived control to keep on trying to go as low as possible, while having the support network in case symptoms arose. This gave me a clear goal. Now, being out of the trial, I continually remind myself that this goal is important. There are trade-offs to be made between the acceptance of a minimal amount of symptoms and OCS dosage. The greater the OCS dosage is, the less asthma-related symptoms there are, but with a greater risk of OCS-related symptoms. The tricky part is that the latter are not experienced in the same way as the disease symptoms, which are often more acute with a direct impact on quality of life. This is especially true in my case, as I tolerate OCS well. In other words, it is easy to alleviate EGPA-related symptoms, including asthma, by increasing OCS dosage, directly increasing my quality of life. The questions I ask myself are: How low in OCS dosage do I want to go, and to what extent do I accept symptoms that do not compromise my quality of life, all while keeping in mind longterm effects of high-dosage OCS? The answers to these questions vary with time and are related to social activities and environment; it is a dynamic threshold.

\section{Physician's Point of View on Clinical Trial Participation with Rare and Serious Chronic Diseases}

There are many advances being made in the field of severe asthma, EGPA, and other chronic inflammatory diseases. New drugs that are specifically directed against a cell type or molecule critically involved in pathogenesis are being developed regularly. For many of these drugs, participation in clinical trials is the only way for patients to access treatment, and there are, in general, a limited number of referral centers that can enroll patients. Most patients depend on their physicians for information about new drugs and their availability, and, in the setting of rare diseases, few physicians are informed themselves. Hence, in many cases, the opportunity for a patient to participate in a clinical trial and access new drugs occurs by chance. Progress is needed in bringing this information to patients and physicians.

One aspect of clinical trials is that patients and physicians meet more regularly than usual, resulting in globally improved management, as background therapy is better adapted to the patient's status at a given time. Careful attention is paid to patient complaints, as they must be captured and recorded for the trial, especially for the relationship between these complaints and the new drug under investigation, the disease itself, or OCS dosing or tapering. In the specific clinical trial mentioned in this paper [7], the main endpoint of the study was disease remission, defined as the absence of disease activity despite the fact that OCS treatment was tapered to a very low dosage. The trial design imposed a very dynamic OCS tapering attitude to compare remission rates between patients treated with the active drug under study versus those receiving placebo. One problem with 
tapering OCS is that patients often experience AEs related to steroid withdrawal that can entice the patient or physician to increase the dosage, either because the symptoms are mistakenly interpreted as reflecting increased disease activity and/or just to feel better again. Enhanced patient and physician awareness of this through clinical trial participation may result in better long-term perception of diseaserelated versus OCS withdrawal-related AEs, and hence tighter control of maintenance OCS therapy.

In line with patient considerations mentioned in the preceding section, it is increasingly recognized that patient well-being, which integrates both disease control and treatment tolerance, should be considered globally when assessing new drugs in complex heterogeneous disorders such as those discussed here. Tools based on patient-reported outcomes are increasingly perceived as a useful approach to assess the global impact of new treatments on health. However, such tools fail to capture the long-term, initially silent, effects of prolonged OCS use that provide a strong motivation for the development of new targeted OCS-sparing agents.

\section{Patient Perspective on Patient Education}

Not every patient should be bombarded with patient information, but, at the same time, there is a real need for precision education based on patient profiles and characteristics. As patient needs and conditions vary over time, it can be characterized as dynamic and individual. This is also affected by the degrees of (in)tolerance of uncertainty. The degree of uncertainty will be greater just after diagnosis than later in the patient journey.

As there will be moments when patients look up information online, it is important to be aware about the differences between, for example, prednisone, prednisolone, and methylprednisolone, etc. Raising this point with patients could be sufficient without going into the details to make them aware that apples should not be compared with pears. Patients should be encouraged to take an active part in their education and hygiene when possible. General goal setting in life, participating in social activities, and doing relaxation exercises and physical activity might help keep a healthy mental status.

\section{CONCLUSION}

Management of chronic inflammatory diseases, such as severe asthma, or of orphan diseases like EGPA, requires a life-long commitment on behalf of both patient and physician to effectively communicate the most pertinent information at a given time during the disease course. For prolonged OCS use, patient education, implementation of optimal preventive measures, and a search for more targeted OCSsparing agents remain the physician's responsibility, notwithstanding the increased access to Web-based information and education for patients. The way that physicians describe the disease course and OCS toxicity to patients impacts patient involvement in dynamic OCS tapering and adherence to treatment, and the way that patients describe their symptoms and global health status to physicians allows for eventual refinement of diagnosis and continuous adaptation of treatment by the latter. With significant advances being made in the pathogenesis and development of targeted drugs that allow OCS-sparing, heightened awareness of ongoing clinical trials and physician-patient communication have become essential for optimal disease management.

\section{ACKNOWLEDGEMENTS}

Funding. AstraZeneca funded the study and the journal's rapid service and open access fees.

Medical Writing and/or Editorial Assistance. Editorial support was provided by JK Associates, Inc., and Michael A. Nissen, ELS, of AstraZeneca. This support was funded by AstraZeneca. 
Authorship. Both named authors meet the International Committee of Medical Journal Editors (ICMJE) criteria for authorship for this article, take responsibility for the integrity of the work as a whole, and have approved this version to be published.

Disclosures. The patient was compensated for time spent in consulting activities for AstraZeneca, but neither the patient nor physician were compensated for authorship work on this manuscript. Dr Roufosse performed consulting activities for GlaxoSmithKline, Knopp Biosciences, and AstraZeneca, and has received honoraria from GlaxoSmithKline and Knopp Biosciences for these services. Mitchell Silva has nothing to disclose.

Compliance with Ethics Guidelines. This article does not contain any new studies with human or animal participants performed by any of the authors.

Data Availability. Data sharing is not applicable to this article as no data sets were generated or analyzed during the current study.

Open Access. This article is distributed under the terms of the Creative Commons Attribution-NonCommercial 4.0 International License (http://creativecommons.org/licenses/ by-nc/4.0/), which permits any noncommercial use, distribution, and reproduction in any medium, provided you give appropriate credit to the original author(s) and the source, provide a link to the Creative Commons license, and indicate if changes were made.

\section{REFERENCES}

1. Costello R, Patel R, Humphreys J, McBeth J, Dixon WG. Patient perceptions of glucocorticoid side effects: a cross-sectional survey of users in an online health community. BMJ Open. 2017;7:e014603.

2. Olajos-Clow J, Costello E, Lougheed MD. Perceived control and quality of life in asthma: impact of asthma education. J Asthma. 2005;42:751-6.

3. Nguyen Y, Guillevin L. Eosinophilic granulomatosis with polyangiitis (Churg-Strauss). Semin Respir Crit Care Med. 2018;39:471-81.

4. Global Initiative for Asthma. Global strategy for asthma management and prevention, 2018. http:// www.ginasthma.org. Accessed Aug 72019.

5. Volmer T, Effenberger T, Trautner C, Buhl R. Consequences of long-term OCS therapy and its side-effects in severe asthma in adults: a focused review of the impact data in the literature. Eur Respir J. 2018;52:1800703.

6. Busse WW. Biological treatments for severe asthma: a major advance in asthma care. Allergol Int. 2019;68:158-66.

7. Wechsler ME, Akuthota P, Jayne D, et al. Mepolizumab or placebo for eosinophilic granulomatosis with polyangiitis. N Engl J Med. 2017;376:1921-32. 